HISPANIA. Revista Española de Historia, 2010, vol. LXX, núm. 236, septiembre-diciembre, págs. 765-786, ISSN: 0018-2141

\title{
¿UNA OPORTUNIDAD PERDIDA? POLÍTICAS DE CONTROL DEMOGRÁFICO EN LA CHINA DE MAO (1949-1976)
}

\author{
DANIEL GOMÁ \\ Universidad de Barcelona
}

RESUMEN: Este trabajo analiza las políticas de control de la natalidad desarrolladas en China durante el periodo maoísta (1949-1976). Partiendo del análisis de fuentes chinas y extranjeras, pretendemos explicar cómo se desarrollaron las diferentes campañas de planificación familiar y cómo éstas se vieron condicionadas positiva y negativamente por las decisiones del dirigente supremo Mao Zedong. Aunque de ellas, sólo la tercera y última tuvo un impacto importante en la lucha contra la superpoblación del país, todas ellas contribuyeron a impulsar la necesidad de hacer frente a este fenómeno y, más importante todavía, sentaron las bases de las políticas antinatalistas que tienen lugar en China actualmente.

Palabras clave: China. Mao Zedong. Demografía. Control de nacimientos. Planificación familiar. Métodos anticonceptivos.

\section{A LOST CHANCE? BIRTH CONTROL POLICIES IN MAO’S China (1949-1976)}

ABSTRACT: This article analyzes the birth control policies in China during the Maoist era (19491976). Considering Chinese and foreign sources, it is explained bow the different campaigns of family planning took place and how these campaigns were subjected positively and negatively to the policies of the supreme leader Mao Zedong. Although only the last one had a real impact on the fight against overpopulation, all campaigns belped to promote the necessity of confronting this phenomenon. More importantly, they laid the foundations of the birth control policies that currently take place in China.

KEY WORDS: China. Mao Zedong. Demography. Birth control. Family planning. Contraceptive methods.

Daniel Gomá es miembro del Departamento de Historia Contemporánea de la Universidad de Barcelona. Dirección para correspondencia: Facultad de Geografía e Historia, Universidad de Barcelona, C/ Montalegre 6, 08001 Barcelona (España).Correo electrónico:dgoma@ub.edu. 
China se convirtió desde tiempos antiguos en la nación más poblada del planeta y en una de las primeras que calculó su población. Con anterioridad al siglo XVIII el país tuvo un crecimiento demográfico muy lento. Conflictos bélicos, epidemias, desastres naturales, etc. frenaron cualquier posibilidad de incremento de la población china. Sin embargo, el largo periodo de estabilidad política y el desarrollo económico que va de 1661 a 1850 aproximadamente fueron el detonante de un crecimiento demográfico brutal que haría que la población china pasase de unos 100 millones a mediados del siglo XVII a alrededor de 420 millones doscientos años más tarde ${ }^{1}$. En dos siglos, la población se había multiplicado por cuatro pero el problema no será este hecho en sí mismo, sino la masa de población que se cuadruplicará. La inestabilidad política y las guerras que sacudieron China entre 1850 y 1949 ralentizaron el aumento de la población pero al mismo tiempo impidieron que se tomasen medidas para poner freno a este crecimiento desbocado. Las consecuencias se verían a partir de la segunda mitad del siglo XX.

Cuando en octubre de 1949 se fundó la República Popular China (RPCh), el país contaba ya con 541 millones. Dos eran los objetivos del nuevo régimen marxista liderado por el Partido Comunista Chino ( $\mathrm{PCCh}$ ), encabezado por Mao Zedong, en lo que se refiere a su política demográfica. El primero era reducir los elevados índices de mortalidad mediante el desarrollo de la sanidad pública y el auge del nivel de vida de los ciudadanos. El segundo objetivo era incrementar la esperanza de vida de estos últimos. En ambos casos los avances fueron notables y, en este sentido, los objetivos se cumplieron con creces. El problema es que dicho éxito no vino acompañado al mismo tiempo de una política firme y estable de control de la natalidad hasta los últimos años de la era de Mao (1949-1976).

Una de las causas de la ausencia de una política antinatalista clara fue, sin duda, la ingente cantidad de desafíos que debía hacer frente el nuevo gobierno comunista para sacar adelante a un país atrasado y pobre, pasando la cuestión demográfica a un segundo plano. Acabar con las elevadas tasas de mortalidad, tanto adulta como infantil, era prioritario. Pero no menos importante fue la acción del propio Mao, quien como líder supremo jugó un papel fundamental en todas las decisiones políticas hasta su fallecimiento en 1976. La visión de Mao respecto al control demográfico varió a lo largo de los años, principalmente en función de los acontecimientos políticos y de las necesidades revolucionarias, y ello tuvo un impacto decisivo en el desarrollo de todos los programas de planificación familiar impulsados por el gobierno chino hasta su muerte.

1 WILL, Pierre-Étienne, «De l'ère des certitudes à la crise du système» en: BERGÈRE, MarieClaire, BIANCO, Lucien y DOMES, Jürgen (eds.), La Chine au XXe siècle. Volume 1: D'une révolution à l'autre (1895-1949), París, Fayard, 1989, págs. 9-43. 
TABLA 1. POBLACIÓN Y TASAS DE NATALIDAD, MORTALIDAD Y FECUNDIDAD EN CHINA (1949-1976)

\begin{tabular}{|c|c|c|c|c|}
\hline Año & $\begin{array}{c}\text { Población } \\
\text { (en millones) }\end{array}$ & $\begin{array}{c}\text { Tasa de } \\
\text { natalidad } \\
(\% o)\end{array}$ & $\begin{array}{c}\text { Tasa de } \\
\text { mortalidad } \\
(\% 0)\end{array}$ & $\begin{array}{c}\text { Tasa de } \\
\text { fecundidad } \\
\text { (hijos } \times \text { mujer) }\end{array}$ \\
\hline 1949 & 541.67 & 36,0 & 20,0 & 6,14 \\
\hline 1950 & 551.96 & 37,0 & 18,0 & 5,81 \\
\hline 1951 & 563.00 & 37,80 & 17,80 & 5,70 \\
\hline 1952 & 574.82 & 37,0 & 17,0 & 6,47 \\
\hline 1953 & 587.96 & 37,0 & 14,0 & 6,05 \\
\hline 1954 & 602.66 & 37,97 & 13,18 & 6,28 \\
\hline 1955 & 614.65 & 32,60 & 12,28 & 6,26 \\
\hline 1956 & 628.28 & 31,90 & 11,40 & 5,85 \\
\hline 1957 & 645.63 & 34,03 & 10,80 & 6,41 \\
\hline 1958 & 659.94 & 29,22 & 11,98 & 5,68 \\
\hline 1959 & 672.07 & 24,78 & 14,59 & 4,30 \\
\hline 1960 & 662.07 & 20,86 & 25,43 & 4,02 \\
\hline 1961 & 658.59 & 18,02 & 14,24 & 3,29 \\
\hline 1962 & 672.95 & 37,01 & 10,02 & 6,02 \\
\hline 1963 & 691.72 & 43,37 & 10,04 & 7,50 \\
\hline 1964 & 704.99 & 39,14 & 11,50 & 6,18 \\
\hline 1965 & 725.38 & 37,88 & 9,50 & 6,08 \\
\hline 1966 & 745.42 & 35,05 & 8,83 & 6,26 \\
\hline 1967 & 763.68 & 33,96 & 8,43 & 5,31 \\
\hline 1968 & 785.34 & 35,59 & 8,21 & 6,45 \\
\hline 1969 & 806.71 & 34,11 & 8,03 & 5,72 \\
\hline 1970 & 829.92 & 33,43 & 7,60 & 5,81 \\
\hline 1971 & 852.29 & 30,65 & 7,32 & 5,44 \\
\hline 1972 & 871.77 & 27,93 & 7,61 & 4,98 \\
\hline 1973 & 892.11 & 27,93 & 7,04 & 4,54 \\
\hline 1974 & 908.59 & 24,82 & 7,34 & 4,17 \\
\hline 1975 & 924.20 & 23,01 & 7,32 & 3,57 \\
\hline 1976 & 937.17 & 19,91 & 7,25 & 3,24 \\
\hline
\end{tabular}

Fuentes: White, Tyrene, China's Longest Campaign: Birth Planning in the People's Republic, 1949-2005, Ithaca, Cornell University Press, 2006; LiANG, Qiusheng y LeE, Che-Fu, "Fertility and Population Policy: An Overview" en: Poston, Dudley L., LeE, Che-Fu, Cheng, Chiung-Fang, Mackibben, Sherry L. y Walther, Carol S. (eds.), Fertility, Family Planning, and Population Policy in China, Nueva York, Routledge, 2006, págs. 7-18.

\section{LA VISIÓN MAOÍSTA DEL CONTROL DEMOGRÁFICO EN LOS PRIMEROS AÑOS DE GOBIERNO COMUNISTA}

La fundación de la RPCh en 1949 marcó el final de un periodo de más de un siglo de inestabilidad interna en el país. El optimismo reinante ante la nueva era en la que estaba entrando China hacía pensar que aquellos 
problemas que le afectaban desde hacía siglos serían resueltos con el nuevo y triunfal espíritu revolucionario. Uno de los problemas era, sin lugar a dudas, el control de la natalidad. Sin embargo, este último no fue incluido entre las prioridades del nuevo gobierno comunista de Pekín en los primeros años de su existencia. A ojos de Mao y de muchos de sus camaradas, el elevado número de habitantes del país no era motivo de preocupación. Al contrario, toda mención al control de la natalidad fue rechazada de plano y se calificó dicha política de reaccionaria, capitalista, imperialista, antihumana y sobre todo de antichina. ${ }^{2}$ Los medios de comunicación chinos llegaban al extremo de denunciar los argumentos de los expertos occidentales partidarios de la planificación familiar, a quienes acusaban de buscar «un medio de exterminar al pueblo chino evitando mancharse las manos con sangre ${ }^{3}$. Tras más de un siglo de crisis y guerras, el país volvía a estar unificado y en paz y el auge de los nacimientos a partir de 1949 era percibido como un signo de recuperación nacional. La China comunista tenía problemas más importantes que resolver: la consolidación política, la restauración del orden social y la reconstrucción económica, todo ello en el marco de un objetivo revolucionario que no era otro que transformar una sociedad agraria y atrasada en una avanzada y socialista. En la búsqueda de este objetivo, el crecimiento de la población era percibido como un arma poderosa de China y en esta época el lema seguido era «cuantos más seamos, más fuertes seremos».

La idea de una amplia población como un elemento positivo del programa de desarrollo económico que se estaba poniendo en marcha queda confirmada con la descalificación del mito maltusiano de que el régimen no podría alimentar a la población si ésta crecía a un ritmo elevado. El propio Mao aseguraba confiado que el crecimiento demográfico nunca causaría problemas a China porque la productividad de las masas después de la revolución sería casi ilimitada. Así, no había dudado en afirmar a finales de 1949:

«Es una buena cosa que China tenga una gran población. Incluso si la población de China se multiplica muchas veces, China será capaz de encontrar una solución; la solución es la producción. El argumento absurdo de los economistas burgueses occidentales como [el inglés Thomas] Malthus en el sentido de que el crecimiento alimentario no podía mantener el ritmo del crecimiento de población no sólo fue refutado en teoría por los marxistas hace ya mucho tiempo sino que se ha demostrado completamente falso ante las realidades [acontecidas] en la Unión Soviética y las áreas liberadas de China después de sus revoluciones... Creemos que la revolución puede cambiarlo todo y que en poco tiempo emergerá una nueva China con una gran población y una gran riqueza de

\footnotetext{
2 Scharping, Thomas, Birth Control in China, 1949-2000: Population Policy and Demographic Development, Londres, RoutledgeCurzon, 2003, pág. 43.

3 Sun Jingzhi, "Suqing dilixue zhong de chong Mei sixiang [Eliminar la ideología americana en el campo de la geografía]" en: Renmin ribao (Diario del Pueblo), 25 de abril de 1952.
} 
productos, donde la vida será abundante y la cultura florecerá. Todas las visiones pesimistas son completamente infundadas ${ }^{4}$.

Este rechazo a las tesis maltusianas, heredado de las teorías del dictador soviético Josef Stalin, radicaba en varios motivos. El primero era su origen extranjero. Las referencias a la posible falta de recursos alimentarios para una población de gran tamaño y en rápido crecimiento fueron rechazadas con el argumento de estar planteadas sin tener un verdadero conocimiento de la realidad de China, donde la cuestión del crecimiento poblacional no había sido motivo de importantes discordias hasta entonces. Por otro lado, menos de un año después de la fundación de la RPCh, este país se había embarcado en un enfrentamiento bélico con EE.UU. en Corea y Mao consideraba que la superioridad poblacional de China sobre la norteamericana, traducida en la mayor presencia de soldados chinos, era una de las claves de la resistencia frente al ejército más poderoso del mundo. Al descartar la implementación de una política antinatalista, Mao, en realidad, no hacía sino heredar la visión demográfica de los líderes chinos del periodo republicano anterior (Sun Yat-Sen y Chiang Kai-shek), quienes habían defendido siempre un crecimiento demográfico elevado como forma de salvaguardar la independencia nacional ${ }^{5}$. El segundo motivo de rechazo respondía a cuestiones internas al régimen. Durante los años de lucha revolucionaria, el PCCh había llevado a cabo una política en principio contradictoria de incitar al auge de la natalidad debido a la alta mortalidad infantil, las enfermedades y las altas tasas de mortalidad en las áreas bajo su control, mientras que al mismo tiempo presionaba a las parejas jóvenes a retrasar el matrimonio y el nacimiento de hijos con el fin de destinar todas las energías a la lucha revolucionaria ${ }^{6}$. No es extraño, por tanto, que una vez logrado el triunfo el Partido fuera un entusiasta de los matrimonios y del auge del número de nacimientos. El nuevo ministerio de Sanidad se apuntó a la campaña mediante una restricción en las políticas de planificación familiar y logró sortear incluso las presiones de la Federación de Mujeres, liderada por la esposa del primer ministro Zhou Enlai, Deng Yingchao. Según las nuevas normas, el acceso a métodos anticonceptivos así como la práctica de abortos y esterilizaciones fue notablemente restringido en el caso de funcionarios, cuadros del Partido, miembros del gobierno, etc. La importación de contraceptivos

4 MAO Zedong, "The Bankruptcy of the Idealist Conception of History", Selected Works of Mao Tse-tung (vol. 4), Pekín, Foreign Languages Press, 1961, págs. 452-455. Es interesante señalar que este capítulo donde se encuentra el fragmento citado era la respuesta de Mao a las críticas del secretario de Estado norteamericano de entonces, Dean Acheson, quien señalaba la gran población china como causa del triunfo comunista en 1949 y de la amenaza que ello suponía para el nuevo régimen si no era capaz de satisfacer las demandas de dicha población.

5 SCHARPING, Thomas, Birth Control in China..., pág. 30.

6 White, Tyrene, China's Longest Campaign: Birth Planning in the People's Republic, 1949-2005, Ithaca, Cornell University Press, 2006, pág. 20. 
fue prohibida ${ }^{7}$. En realidad, toda la sociedad se vio afectada y el crecimiento poblacional se aceleró.

\section{LA PRIMERA CAMPAÑA DE PLANIFICACIÓN FAMILIAR}

No obstante, la presión de algunos dirigentes del Partido, como Deng Yingchao, se hizo más evidente a partir de 1953. Los cuadros con más hijos tenían mayores problemas para combinar trabajo y hogar y ello afectaba a su rendimiento. La esposa de Zhou Enlai no dudó en utilizar sus contactos para denunciar este problema. En una carta destinada a ella en 1953, el viceprimer ministro Deng Xiaoping le respondía señalando que «creo que es necesario y beneficioso promover de manera efectiva el uso de la contracepción» ${ }^{8}$. Poco después, en agosto de 1953, el Consejo de Estado (nombre oficial del gobierno chino) decidió plantear la necesidad de imponer restricciones en los nacimientos mediante una directiva interna dirigida al ministerio de Sanidad. Nacía así la primera política de control de la natalidad o planificación familiar (jibua shengyu) de China aunque todavía tardaría un tiempo en entrar en vigor. De manera no oficial se decretaron las primeras medidas, consistentes en el levantamiento de ciertas restricciones a la hora de acceder a anticonceptivos y la defensa del control de la natalidad entre el personal médico de todo el país 9 .

Las razones de esta discreción radicaban en la amenaza que suponían los opositores al control de la natalidad y a la importante influencia que ejercían en el seno del régimen. Aunque la resistencia a esta política no tardó en hacer acto de aparición, los opositores tuvieron que retirarse temporalmente ante los nuevos acontecimientos. La sorpresa cundió en el gobierno y el PCCh cuando a mediados de 1954 se hicieron públicos los resultados del primer censo nacional realizado el año anterior. Durante los primeros años de la RPCh, el régimen comunista había empleado con frecuencia la cifra de los «500 millones» para demostrar el poder de la nueva China. Muchos de sus dirigentes pensaban que el número era inferior, tal vez 475 millones, pero a partir de 1951-1952 cundió la preocupación por que la cifra real fuera mayor de la prevista. Así, el censo de 1953 demostró la dolorosa realidad: el país contaba con 583 millones de habi-

7 AIRD, John S., Slaughter of the Innocents: Coercive Birth control in China, Washington D.C., The AIE Press, 1990, pág. 20.

8 CA, R., Hu, W. y ZEI, J., Demography of a Century, Beijing, Beijing Arts and Literature Publishers, 1999, págs. 112-113.

9 Estas medidas no fueron hechas públicas hasta 1957. Véase el editorial de Renmin ribao del 5 de marzo de 1957. El texto en cuestión, titulado «Ejercer un control de la natalidad apropiado», fue reproducido en Zhongguo renkou nianjian, 1985 [Anuario de la población china, 1985], Pekín, Zhongguo renkou chubanshe, 1985, págs. 10-11. 
tantes sin contar Taiwán ${ }^{10}$. Con la llegada de la paz y la estabilidad interna, el crecimiento demográfico se había disparado.

Esta situación suponía un grave problema. A las necesidades básicas de la creciente población (educación, sanidad, etc.) se sumaba el hecho de que el plan de modernización de la nación no podría dar empleo a todos sus habitantes en un futuro, especialmente si su número crecía a un fuerte ritmo. Los objetivos del Primer Plan Quinquenal (1953-1957), esto es, la modernización agrícola e industrial y el desarrollo científico, educativo y sanitario, podían quedar seriamente comprometidos si no se tomaban medidas serias porque se habían hecho calculando un número de habitantes claramente inferior al que mostraban los datos del censo. La promesa de una mejora sustancial de las condiciones de vida de los chinos, repetida una y otra vez por el PCCh, podía quedar en entredicho si no se actuaba con rapidez.

Por ello, el gobierno chino se apuntó a las medidas antinatalistas y en julio de 1954 se hicieron públicas las primeras propuestas oficiales con el fin de someterlas a votación dentro de la cúpula dirigente china. La primera muestra pública a favor del control de la natalidad en la historia de la RPCh fue un discurso del diputado Shao Lize en el marco del Congreso Nacional Popular a finales del verano de 1954. Intelectual no afiliado al PCCh pero simpatizante de izquierdas, Shao era un defensor de la planificación familiar desde hacía décadas. En su discurso señaló que «en nuestro país podemos desestimar la cuestión del aborto pero el conocimiento sanitario sobre contracepción debe ser propagado. Además, instrucciones prácticas, medios necesarios y recursos materiales deben ser proveídos» ${ }^{11}$. Prueba de la importancia de su discurso es que fue impreso en el número del mismo día del Diario del Pueblo, el principal periódico del régimen chino. El propio vicepresidente del país y segunda autoridad del PCCh después de Mao, Liu Shaoqi, apoyó la necesidad de promover un control sobre los nacimientos en un simposio celebrado en la capital china en diciembre de 1954 y propuso que un debate tuviera lugar en el seno del Partido a este respecto ${ }^{12}$. A partir de ahora, los términos «planificación familiar» y «control de la población» (renkou kongzhi) pasaron a ser utilizados de forma cada vez más frecuente por sus dirigentes $^{13}$. A finales de 1954 entraba en vigor oficialmente la primera política de control de la natalidad de la historia de China. En los meses siguientes, una comisión formada por representantes de varios ministerios, encabezados por el de Sanidad, inició un estudio sobre las medidas que se deberían tomar.

10 Los censos demográficos hasta la fecha en la RPCh han sido los siguientes: 1953 (con una población de 583 millones), 1964 (694 millones), 1982 (1008 millones), 1990 (1133 millones) y 2000 (1265 millones).

11 "Deputy Shao Li-tzu Speaks on Birth Control to the National People's Congress", South China Morning Post, 2 de noviembre de 1954.

12 LIU Shaoqi, "Tichang jieyu [Promover el control de nacimientos]", Jiangkang Bao (Revista de salud), Pekín, 20 de noviembre de 1983, pág. 1.

13 White, Tyrene, China's Longest Campaign..., pág. 32. 
Esta primera campaña se centró principalmente en animar a las parejas a planear con antelación el número de hijos y a tomar medidas con el fin de evitar embarazos no deseados ${ }^{14}$. Una forma era retrasando la edad de matrimonio y la otra, espaciar el tiempo entre el nacimiento de un hijo y otro, recurriendo al mismo tiempo al uso de anticonceptivos ${ }^{15}$. Para dirigir la campaña se creó un equipo conocido oficialmente con el nombre de Nacimientos Planificados y liderado por Chen Muhua ${ }^{16}$. Durante la celebración del VIII Congreso del PCCh (septiembre de 1956), numerosos dirigentes abogaron por intensificar las medidas antinatalistas. En su informe, Zhou Enlai señaló que «una medida adecuada de control de natalidad es deseable» ${ }^{17}$. Sin embargo, dentro del régimen había otros que se resistían a estas medidas. Chen Boda, uno de los principales teóricos del Partido, no había dudado en declarar en febrero de 1956 que «no hay signos de sobrepoblación en China... China puede proveer espacio al menos para otros 600 millones de personas ${ }^{18}$. Con todo, el apoyo de Mao, aparentemente convencido de los peligros de un crecimiento rápido de la población, resultó fundamental para que las primeras medidas de control de la natalidad salieran adelante. Parece ser que los primeros problemas de suministro alimentario habían hecho su aparición en 1955 y el máximo líder señaló que «aquellas áreas con densidades elevadas de población deberían promover el control de nacimientos» ${ }^{19}$. Poco después llegó a advertir de que el crecimiento incontrolado de la población podía llevar a la extinción del pueblo chino y que era necesario un programa de planificación familiar que durara diez años pero este último debía tener lugar «en sitios seleccionados [previamente] y después extendido paso a paso hasta que la planificación familiar gradualmente se convierta en universal $»^{20}$. El control de la natalidad pasó a ser una política oficial del Partido y la campaña de planificación familiar se intensificó a partir del otoño de 1956.

14 Las diferentes campañas de planificación familiar durante la época de Mao no afectaron a las minorías nacionales. Estas últimas, que representan menos del $10 \%$ de la población total de China, quedaron exentas de cumplir las directrices del Partido en cuanto a control de nacimientos, que se aplicó sólo a la mayoría Han.

15 White, Tyrene, China's Longest Campaign..., pág. 35.

16 Liang, Qiusheng and LeE, Che-Fu, "Fertility and Population Policy: An Overview" en: Poston, Dudley L., Lee, Che-Fu, Cheng, Chiung-Fang, MckibBen, Sherry L. y Walther, Carold S. (eds.), Fertility, Family Planning, and Population Policy in China, Nueva York, Routledge, 2006, págs. 7-18.

17 Zou Ping, "The Review and Discussion of Changes in China's Population Policy during the Early Years of the People's Republic”, Population Research, 4, 4 (1987), págs. 1-8.

18 Tien, H. Yuan, China's Population Struggle: Demographic Decisions of the People's Republic, 1949-1969, Columbus, Ohio State University Press, 1973, pág. 185.

19 "Woguo de jihua shenyu gongzuo [Trabajo de Planificación Familiar de Nuestro País]", Guangbui de changjiu (Logros gloriosos), volumen 2, Pekín, Renmin chubanshe, 1984, págs. 446-486.

20 MAO Zedong, "Be Activists in Promoting the Revolution", Selected Works of Mao Tse-tung, volumen 5, Pekín, Foreign Languages Press, 1977, págs. 485-489. 


\section{EL FRACASO DE LA PRIMERA CAMPAÑA}

Demasiado breve en el tiempo, esta primera campaña fracasó en primer lugar por la ausencia de una preparación previa, destacando la falta de información y de personal cualificado, la escasez de anticonceptivos y la resistencia entre médicos y demás personal sanitario. El organismo responsable era el ministerio de Sanidad y, en concreto, su departamento de Maternidad y Cuidado Infantil pero el sistema sanitario chino no estaba preparado para llevar a cabo una campaña seria de planificación familiar y en realidad la responsabilidad de llevar a cabo esta última recayó principalmente en los profesionales médicos y no en los cuadros del PCCh. En la práctica, la campaña se redujo en muchos casos a un trabajo de información donde se empezó a propagar la necesidad de retrasar la edad de matrimonio y a aconsejar sobre los métodos de anticoncepción ${ }^{21}$. Aunque las restricciones para abortar y esterilizarse se relajaron notablemente (hasta mayo de 1957 sólo las mujeres con cuatro o más hijos tenían derecho a abortar) y se incrementó el suministro de anticonceptivos, ya fueran importados o fabricados en el país (en 1957 China produjo 45 millones de preservativos), la respuesta social fue limitada ${ }^{22}$. Desde el punto de vista político, lo que sucedió es que hubo una ausencia de organización para trasladar las directivas antinatalistas del Partido a la realidad, debido en gran medida al hecho de que la dirección política de la misma recayó en la administración provincial y local, donde la falta de medios y de instrucciones claras fue la tónica general.

La campaña se llevó a cabo de manera lenta y de forma descoordinada. Así, por ejemplo, algunas provincias la empezaron más tarde que otras. El objetivo fueron ante todo las zonas urbanas, dejándose de lado las áreas rurales, más pobladas. Las ciudades, con sus espacios más limitados, eran objetivo preferente porque eran consideradas zonas de mayor riesgo ante la sobrepoblación (problemas de sanidad, higiene, etc. $)^{23}$. A todo esto se le unían las resistencias que procedían de la sociedad. Por ejemplo, la medida de retrasar la edad de matrimonio no era bien vista entre las parejas y mucho menos, el aborto. Todavía persistían mentalidades pasadas, destacando el enorme poder de las suegras, consideradas un modelo a seguir por las nueras incluso en el ámbito de la reproducción, esto es, el modelo de la familia extensa como signo de buena fortuna. La venta de preservativos fue muy baja debido al poco interés de las parejas en limitar el número de hijos.

21 Scharping, Thomas, Birth Control in China..., pág. 46. El aborto seguía siendo un tema tabú en la sociedad china e incluso buena parte de los dirigentes del régimen comunista se oponía a su legalización plena. De hecho, en las dos décadas anteriores a 1949, el PCCh había penalizado el aborto en los territorios bajo su control. En los primeros años de la RPCh, la autorización para abortar fue siempre muy limitada y sujeta a casos especiales.

22 BANISTER, Judith, China's Changing Population, Stanford, Stanford University Press, 1987, pág. 148.

23 Peng Xizhao, Demographic Transition: Fertility Trends in China since 1954. Oxford, Clarendon Press, 1991, pág. 27. 
Por otro lado, la primera campaña de planificación familiar tenía unos objetivos claramente políticos y económicos y no sociales. Un crecimiento demográfico demasiado rápido podía hacer fracasar los objetivos del gobierno. Por ello, dicha política estaba sujeta a crítica en caso de alterarse los objetivos políticos y económicos. En 1957 la situación política de China se vio alterada con la campaña de las Cien Flores dirigida contra los intelectuales. Entre las consecuencias de esta campaña, que vino seguida poco después del Gran Salto Adelante, la primera gran campaña de utopía maoísta (lanzada a mediados de 1958), estuvo la crítica de las políticas antinatalistas. Los miembros del gobierno partidarios de las mismas tuvieron que hacer una retirada forzosa. Aquellos intelectuales partidarios del control demográfico fueron tachados de reaccionarios. Sólo en 1957 había dedicados al estudio de esta cuestión casi un centenar de especialistas de diferentes disciplinas ${ }^{24}$. La figura principal en estos años fue Ma Yinchu, economista y presidente de la Universidad de Pekín y autor de la obra de referencia Nueva Teoría de la población (1957), donde advertía que la superpoblación podía ralentizar la acumulación de capital y la industrialización del país. Por ello, abogaba por incrementar la producción agrícola a la vez que se controlaba el rápido crecimiento demográfico, calificado como el «problema de población» ${ }^{25}$. Para Ma, la economía socialista planificada debía incluir la planificación familiar como parte esencial de la misma.

Lo que Ma y otros estudiosos no entendieron es que el Gran Salto Adelante alteró incluso la defensa que el propio Mao había hecho del control demográfico hasta la fecha. En un momento en que el líder chino regresaba a una posición de ortodoxia marxista, el principal argumento de Ma y demás defensores de la planificación familiar fue negar la doctrina marxista según la cual una sociedad socialista carece de problemas de población. Además, en un momento en que el objetivo era el desarrollo industrial a partir de los beneficios de un incremento de la producción agrícola (incremento que después se demostró falso y conllevó consecuencias trágicas), el optimismo llevó a que el propio Mao viera en la producción agrícola la forma de alimentar a una población cada vez más numerosa. Uno de los eslóganes de la época era «iuna boca más que alimentar son dos brazos que trabajan!» ${ }^{26}$. El dirigente chino no dudó en señalar que «a más gente... más intenso el fervor y más grande la energía». Incluso la pobreza de China tenía un aspecto positivo porque «les convertía [a los pobres] en mejores revolucionarios y defensores del cambio» ${ }^{27}$. A su juicio, el entusiasmo revolucionario de las masas conllevaría un aumento de la producción, supe-

24 LIANG, Qiusheng y LEE, Che-Fu Lee, "Fertility and Population Policy: An Overview...", pág. 10.

25 Ma Yinchu, The New Population Theory, Changchun, Jilin People's Publishers, 1997.

26 BIANCO, Lucien, «Croissance démographique et politique antinataliste» en: BERGÈRE, Marie-Claire, BIANCO, Lucien y Domes, Jürgen (eds.), La Chine au XXe siècle. Volume 2: De 1949 à aujourd'bui, París, Fayard, 1990, págs. 121-148.

27 “Chairman Mao Tse-tung's Article for 'Red Flag”, South China Morning Post, 4 de junio de 1958. 
rior incluso a la necesaria para alimentar a toda la población. La elevada población era un factor decisivo para el triunfo del Gran Salto Adelante y no debía ser un problema a la larga. El propio Mao no dudaba en considerar que el control de la natalidad se acabaría implantando por sí solo con el paso del tiempo. A este respecto declaró:

«Nuestra visión sobre la población debería cambiar. En el pasado dije que podíamos tratar con 800 millones [de personas]. Ahora creo que mil millones no serían motivo de alarma. Ello no sería recomendable para la gente con muchos hijos. Cuando el nivel de educación [del pueblo] se incremente, [el pueblo] realmente practicará el control de natalidad» 28 .

Todo el país se lanzó a apoyar la utopía maoísta. Los datos sobre la producción agrícola, cada vez más inflados y falseados por unos funcionarios temerosos de las consecuencias en caso de suministrar información negativa, llevaron a una situación de optimismo ante el futuro que esperaba a China. La consecuencia fue el abandono del control de la natalidad como política del gobierno y la prensa dejó de informar sobre el tema, al mismo tiempo que la producción de anticonceptivos disminuía sensiblemente ${ }^{29}$. Los defensores acérrimos de la planificación familiar fueron denunciados como «derechistas» y la mayoría de ellos (caso de Chen Changheng y Chen Da, «padres» de la demografía china) optó por una retirada discreta. Sólo Ma Yinchu persistió en sus ideas y fue purgado en marzo de 1960. Muchos dirigentes del Partido, incluidos los hasta entonces impulsores del control de la natalidad (Liu Shaoqi, Zhou Enlai, Deng Xiaoping, etc.) se unieron al coro de voces que veían en el crecimiento de la población una ventaja para el Gran Salto Adelante. En este sentido, el secretario de la Liga de las Juventudes Comunistas (juventudes del PCCh) Hu Yaobang afirmó que «una gran población significa gran poder humano... La fuerza de 600 millones de personas libres es decenas de miles de veces más fuerte que una explosión nuclear. Dicha fuerza es capaz de crear maravillas que nuestros enemigos no pueden ni imaginar» ${ }^{30}$.

La euforia general no tardó en extinguirse. A partir de 1959, los problemas de suministro alimentario hicieron su aparición. Las consecuencias del desastre del Gran Salto Adelante tuvieron, curiosamente, un impacto brutal y negativo en el crecimiento demográfico chino aunque debido a circunstancias trágicas. A pesar de que seguimos sin saber la cifra exacta debido a que las autoridades oficiales chinas se niegan todavía hoy a dar información precisa al respecto, se

28 "Talks at Beidaihe Conference, August 17, 1958", en: MACFARQuHar, Roderick, CHEeK, Timothy and Wu, Eugene (eds.), The Secret Speeches of Chairman Mao: From the Hundred Flowers to the Great Leap Forward, Cambridge, Harvard University Press, 1989, págs. 397-441.

29 AIRD, John S., Slaughter of the Innocents..., pág. 24.

30 "Hu Yaobang: Man is the Determining Factor in Our Great Undertakings", en: South China Morning Post, 9 de mayo de 1958. 
acepta que entre treinta y cuarenta millones de personas fallecieron de inanición o como consecuencia de otras malas prácticas derivadas de esta absurda campaña de industrialización forzosa y rápida impulsada por Mao en su sueño utópico de convertir a China en una de las principales potencias industriales en menos de una generación. Por primera y única vez desde 1949, China experimentó un crecimiento negativo de la población en 1960-1961, en parte consecuencia también de una caída brusca en el número de nacimientos que no empezó a recuperarse hasta 1962. Así, de una población de 672 millones de personas en 1959 se pasó a 662 un año más tarde y 658,5 en 1961. Si nos fijamos bien en los índices de la tabla 1, veremos que la tasa de mortalidad en esta época superó el 25\%o, una cifra incluso superior a la que existía en los primeros años de la RPCh ${ }^{31}$.

No obstante, la cuestión de la sobrepoblación seguía estando encima de la mesa de las autoridades. El descalabro del Gran Salto Adelante vino seguido de un incremento significativo de la natalidad, alcanzándose una tasa de 37,01\%o, $43,37 \%$ y 39,14\%o en 1962, 1963 y 1964 respectivamente, las dos últimas de ellas, las más altas en la historia de la RPCh. Si a ello añadimos un progresivo descenso de la tasa de mortalidad en estos años hasta niveles cercanos a los de antes del Gran Salto Adelante, no es sorprendente que el censo nacional de 1964, establecido en 694 millones, causara una gran preocupación entre los dirigentes del régimen comunista. Los datos del segundo censo nacional señalaban claramente que el país había crecido en unos ciento cincuenta millones de personas en el plazo de una década y media.

El importante crecimiento de la población entre 1949 y 1964 obedece, sin embargo, a varias razones. La primera es el mantenimiento de la tasa de fecundidad de la mujer china, que pasa de 6,14 a 6,18. En sí, el incremento es muy pequeño pero pone de manifiesto que las políticas de control de la natalidad desarrolladas durante la primera década y media fueron un sonoro fracaso pese a las acciones del gobierno. Si exceptuamos el periodo 1959-1961, los datos indican una tasa anual de 6 hijos por mujer. En una sociedad donde las estructuras familiares seguían teniendo un carácter muy tradicional y el alto número de hijos era visto como un factor positivo, los intentos del PCCh de reducir dicho número tendrían un impacto muy limitado.

Otro de los factores fundamentales para entender el crecimiento demográfico fue la reducción de la tasa de mortalidad. En 1949 era de 20,0\%o pero década y media más tarde se había reducido a menos de la mitad, 9,5\%o. A ello hay que añadir la tasa de mortalidad infantil que también se redujo de manera significativa, alcanzando alrededor del $86 \%$ en 1964 , un éxito sin

31 Estas cifras son las oficiales del régimen comunista chino. Fuentes occidentales señalan que en realidad los datos referidos a la tasa de mortalidad, tasa de mortalidad infantil y el índice de nacimientos son mucho mayores. Para una versión diferente de estos datos, véase BANISTER, Judith, China's Changing Population..., pág. 352. 
paliativos si tenemos en cuenta que la cifra en 1949-1950 probablemente se situaba entre 180 y $200 \% 0^{32}$. Por último, si incluimos una esperanza de vida cercana a los 60 años, muy superior a los 40-45 de 1949, es inevitable que hubiera un crecimiento poblacional significativo.

\section{El SEgUNDO INTENTO «FALLIDO»}

El problema principal, como vemos, no es otro que la elevada tasa de fecundidad. Después de los años del bajón demográfico ocasionado por el Gran Salto Adelante, estalló un «baby boom», iniciado a partir de 1962 y que se prolongaría durante el resto de la década. Entre 1962 y 1965 la tasa de la natalidad osciló entre el 37 y el $43 \%$ y la tasa de fecundidad se situó entre 6 y 7,50 hijos por mujer. Ello es debido, en gran medida, a la necesidad de las familias chinas de compensar los nacimientos que no tuvieron lugar en los años 1960-1961 debido a la hambruna general y al auge de los matrimonios, cuyo número había descendido en esos años.

Todos estos aspectos no se les escapaban al gobierno de Zhou Enlai. Consciente de la realidad del problema, este último había promovido ya desde finales de 1962 una nueva campaña de planificación familiar fundamentada en dos grandes ejes: la defensa de un matrimonio más tardío y el desarrollo de la familia con dos hijos únicamente como modelo ideal de familia ${ }^{33}$. Aprendiendo de los errores del pasado, esta campaña estuvo mejor preparada y bajo mayor control por parte de las autoridades. El gobierno central tuvo un papel más activo y hubo una mayor implicación de los cuadros del Partido, sobre quienes recaía la responsabilidad principal. Estos últimos debían practicar su propio control de la natalidad como ejemplo para los demás. Asimismo, hubo una mayor especialización de los profesionales médicos que participaban en la campaña.

La nueva directiva señalaba que la planificación familiar era necesaria para la construcción de la nación socialista ya que, pese a ser el nacimiento principalmente un asunto personal y privado, el Estado también se veía afectado porque debía suministrar a los niños, entre otros aspectos, educación y sanidad y asegurar su alimentación. Prueba de la importancia que concedía Pekín al control de la natalidad fue la creación en 1964 de la Comisión de Nacimientos Planificados (dirigida por Zhou Rongxin, un estrecho colaborador de Zhou

32 Los datos respectivos a la tasa de mortalidad infantil no son concluyentes porque no se hicieron estudios detallados con anterioridad a la fundación de la RPCh. Por otro lado, las cifras de época inmediatamente posterior no son tampoco fidedignas y el propio régimen comunista no se mostró muy interesado en publicarlas. En este sentido, Judith Banister otorga unas cifras interesantes partiendo del año 1953 (con una tasa de mortalidad infantil del 175\%o) hasta 1984 (en torno al 48-50\%o). Véase BANISTER, Judith, China's Changing Population..., pág. 352.

33 WHITE, Tyrene, China's Longest Campaign..., pág. 52. 
Enlai), integrada dentro del organigrama del gobierno, y se fundaron subcomisiones vinculadas a aquella a nivel provincial y local ${ }^{34}$.

Impulsada bajo una directriz con el singular título de Promocionando entusiásticamente la planificación de nacimientos, el objetivo final de la campaña no era otro que, en palabras de Zhou Enlai, "promover [el control de la natalidad] en las ciudades y las zonas rurales densamente pobladas, y que haya un control apropiado de la tasa de crecimiento poblacional de manera que el problema de los nacimientos cambie gradualmente de un estado de anarquía a uno de planificación» ${ }^{35}$. El deseo del gobierno era limitar el tamaño de la familia china. Con este fin, la propaganda se encargaba de señalar que dos hijos como máximo eran lo más beneficioso para la pareja y que ésta debía espaciar el nacimiento de los hijos (entre 3 y 5 años a ser posible). Un tercer hijo era poco recomendable y sólo aconsejable si las circunstancia familiares así lo permitían ${ }^{36}$. Un slogan surgido en esta época y muy repetido era «uno [niño] no es poco, dos es adecuado y tres es demasiado» (yige bushao, liangge zhenghao, sange duole). El otro aspecto importante era retrasar la edad de matrimonio explicando que una mayor madurez mejoraba la crianza de los hijos y la relación de pareja. Asimismo, se advertía que tener hijos a una edad temprana podía ocasionar problemas en el recién nacido y en la propia salud de la madre. Al mismo tiempo, se tomaron medidas como la autorización de la venta de anticonceptivos importados del extranjero y la adopción de políticas para hacer más accesibles los abortos y las esterilizaciones ${ }^{37}$.

Esta campaña, mucho más seria que la anterior, buscaba cambiar una serie de hábitos fuertemente implantados en la sociedad china y por ello tenía unos objetivos más a largo plazo. Un mayor presupuesto y la mejor organización de la campaña contribuyeron a que ésta cosechara algunos éxitos. El uso mayor de métodos anticonceptivos, gratuitos en la mayoría de los casos a partir de 1963 , fue una de las claves. Por primera vez se llevaron a cabo inserciones de dispositivos intrauterinos (DIU) y nuevas técnicas en el caso de los abortos contribuyeron a un ligero descenso de la tasa de natalidad. La prueba más obvia fue en las ciudades y la fertilidad urbana en cada una de las provincias había disminuido de manera clara a mediados de la década ${ }^{38}$. Por otro lado, la campaña se caracterizó también por la puesta en marcha por parte de las autoridades de las primeras medidas serias de disuasión. A diferencia de la primera campaña, basada más en el uso de la propaganda, el gobierno chino se decantó por incluir acciones más contundentes con el fin de asegurarse éxitos en su política antina-

34 LIANG, Qiusheng and LEE, Che-Fu, "Fertility and Population Policy...", pág. 11.

35 "Zhonggong zhongyang guowuyuan guanyu renzhen tichang jihua shengyude zhishi [Instrucciones del Comité Central y el Consejo de Estado sobre la promoción entusiástica de la planificación de nacimientos]”, Pekín, 1962. Citado en: Zhongguo renkou nianjian, 1985, pág. 14.

36 AIRD, John S., Slaughter of the Innocents..., pág. 25.

37 CHU, Leonard L., Planned Birth Campaigns in China, Honolulu, East-West Center, 1978, pág. 18.

38 Dicho descenso no fue hecho público con datos serios hasta comienzos de los años ochenta. 
talista. Por ejemplo, se eliminaron los subsidios por nacimiento para aquellas familias que ya tenían tres o más hijos y se restringieron las oportunidades de acceso a la universidad de aquellas parejas que se habían casado demasiado jóvenes ${ }^{39}$.

Sin embargo, no todos los objetivos se consiguieron. Los problemas fueron principalmente tres. Primero, dicha campaña tuvo un éxito parcial en las áreas urbanas pero ninguno en las rurales (donde el número de hijos ha sido históricamente mayor) pese al aumento de la propaganda, las mejoras sanitarias y la mayor presencia de médicos. El descenso de la tasa de fertilidad entre la población urbana a partir de 1963 quedó disimulado por el mantenimiento de las elevadas cifras del campo. La población rural representaba en esta época alrededor del $80 \%$ del total de la RPCh. En segundo lugar, el peso de la tradición seguía siendo importante y aunque tener menos hijos era visto de manera más positiva, ello tenía también unas implicaciones culturales importantes. En la sociedad china los hijos varones han sido históricamente más valorados que las hijas ya que aseguran un cuidado de los padres en la vejez (los progenitores viven con el hijo mientras que la hija se casa y se traslada a casa del marido) y garantizan el culto a los ancestros. Por ello, el deseo de muchas parejas en esta época seguía siendo tener varones y el tener una familia numerosa aseguraba normalmente una descendencia masculina. En cambio, el limitar a dos el número de vástagos hacía que los riesgos de tener únicamente hijas fuera mayor y, por tanto, objeto de rechazo. En la práctica, un alto número de parejas optaban por poner fin a los embarazos cuando tenían un hijo varón. ${ }^{40}$ Por último, un gran problema resultó ser el intento de promover la vasectomía, un procedimiento más simple y menos costoso que el DIU y el aborto, y que acabó siendo un fracaso estrepitoso debido a las resistencias de los hombres chinos, poco proclives a aceptar la esterilización. No es extraño, por tanto, que la disminución de la tasa de nacimientos fuera limitada y pasara de una media en torno al 40\%o en el periodo $1962-1964$ al 36\%o en $1965-1966$ pese a los intentos del gobierno de disminuir estas cifras por debajo del $30 \%$.

Debido a sus objetivos a más largo plazo, no eran de esperar grandes resultados en los primeros años. De hecho, la campaña estaba diseñada para que durase unos diez años y, una vez analizados los resultados, tomar decisiones más drásticas y corregir errores. El problema era que un plazo tan largo tenía sus riesgos en caso de inestabilidad política y fueron precisamente las circunstancias políticas de la segunda mitad de los sesenta las que provocaron el incumplimiento de los objetivos fijados por el gobierno. Como en el pasado con

39 Peng Xizhao, Demographic Transition..., pág. 31.

40 También había casos en que las parejas deseaban tener una niña cuando tenían sólo hijos varones. En la sociedad china juega un papel importante la idea del yin y el yang, opuestos complementarios que funcionan como un todo. En las relaciones humanas el yin está asociado al sexo femenino y el yang al masculino. Dicho de otro modo, el tener un hijo y una hija es lo mejor para una pareja ya que ello supone la perfección. 
el Gran Salto Adelante, la política de planificación familiar se vio profundamente afectada por el estallido de la Gran Revolución Cultural a mediados de 1966. Con un aparato administrativo completamente desestabilizado y un país sumido en el caos, la segunda campaña de control de la natalidad llegó a su fin.

\section{LOS PRIMEROS ÉXITOS IMPORTANTES: LA GRAN CAMPAÑA DE PLANIFICACIÓN FAMILIAR DE FINALES DEL MAOÍSMO}

El caos político, económico y social en China entre 1966 y 1969 implicó la suspensión de toda actividad relacionada con el control demográfico. La situación empeoró con el auge del número de matrimonios jóvenes y de embarazos, despertando la alarma entre los dirigentes del país. Sin embargo, no fue hasta julio de 1971, una vez pasadas las tensiones más graves de la Revolución Cultural, que el gobierno de Zhou Enlai puso en marcha una nueva campaña de planificación familiar ${ }^{41}$. Esta nueva campaña, que se prolongaría más allá de 1976, fue la más importante de todas las que tuvieron lugar en la era maoísta. Más seria tanto en planteamientos como en objetivos, estaba mucho mejor organizada que las anteriores y contaba con el respaldo firme de los principales dirigentes, Mao incluido. Este último llegó a escribir en un documento interno del Partido que «[el crecimiento de] la población debe ser objeto de control por todos los medios [posibles]» ${ }^{42}$. A lo largo de la primera mitad de los setenta, el gran líder chino declaró en diversas ocasiones la necesidad de controlar el crecimiento demográfico, venciéndose de esta forma las reticencias de algunos sectores del $\mathrm{PCCh}^{43}$. Asimismo, la planificación familiar entró a formar parte definitivamente de los objetivos incluidos en los planes quinquenales que tendrían lugar en esta década ${ }^{44}$. En junio de 1973 se creaba el Grupo Dirigente de Planificación Familiar (que reemplazaba a la Comisión de Nacimientos Planificados abolida durante la Revolución Cultural) y que dirigía un político en ascenso, Hua Guofeng ${ }^{45}$. Al igual que su predecesora, este organismo estaba bajo supervisión del gobierno central. Al mismo tiempo, se reorganizaron los diferentes comités encargados de llevar a cabo la campaña, donde los cuadros del

41 Algunas provincias habían iniciado por su cuenta campañas de planificación familiar ya en 1970. Este fue el caso, por ejemplo, de Guangdong (Cantón), Hunan y Jiangsu.

42 CA, R., Hu, W. and ZEI, J., Demography of a Century..., pág. 166.

43 White, Tyrene, China's Longest Campaign..., pág. 61. 1975.

44 CHu, Leonard L., Planned Birth Campaigns in China..., pág. 25. El primero fue el de 1971-

45 Hua Guofeng fue el sucesor de Mao al frente de China tras la muerte de éste en 1976 pero fue progresivamente apartado del poder por Deng Xiaoping a partir de 1978, desapareciendo de la escena política china a comienzos de los años ochenta. Hua fue un firme partidario de la política de planificación familiar e impulsó durante su breve mandato medidas más restrictivas en este ámbito. En 1975 fue sustituido al frente del Grupo Dirigente de Planificación Familiar por Wu Guixian, candidata al Politburó del PCCh. 
Partido jugaban un papel fundamental. La planificación familiar había pasado a ser una tarea política. Aunque parte de la iniciativa se dejó en manos de las provincias, donde algunas de las medidas podían variar de manera puntual (por ejemplo, la edad ideal de matrimonio), se fijaron desde el gobierno central objetivos anuales.

Conocida como wan xi shao, la nueva campaña buscaba recalcar la necesidad de un matrimonio más tardío (wan), un mayor intervalo en los nacimientos $(x i)$ y menos hijos $(s h a 0)^{46}$. El slogan de «uno [niño] no es poco, dos es adecuado y tres es demasiado» de los años sesenta fue recuperado para animar a las parejas a tener dos hijos como máximo. Por otro lado, se impulsó la creación de organismos vinculados a este proyecto como equipos que informaban, asesoraban y recomendaban a la población sobre la necesidad de frenar el crecimiento poblacional. Estos equipos se encontraban en todos los niveles dentro de la comunidad, ya fuera rural o urbana, e incluía los lugares de trabajo (fábricas, factorías, empresas), vecindarios y comunas populares, donde se formó por orden del gobierno una «red de trabajo de planificación de nacimientos» (jibua shengyu gongzuo wang) ${ }^{47}$. Ello vino acompañado de una mejor información sobre el tema a través de los medios de comunicación (periódicos, revistas femeninas, televisión, radio) o de las acciones de propaganda (posters, películas, conferencias, mítines, etc.). Especialmente importante fue la labor de los cuadros y los médicos rurales, encargados de romper tabúes y la resistencia campesina a la limitación de la descendencia. Para ello, se dotó de mayor presupuesto la campaña en el ámbito rural y se modernizó progresivamente la red sanitaria en el campo $^{48}$. Esto demuestra que el Partido y el gobierno se habían puesto finalmente al frente de la política de control de la natalidad.

Menos visible fue la aparición de una práctica coercitiva por parte del régimen y heredera de las primeras medidas de disuasión impulsadas durante la campaña de los años sesenta. Aunque el gobierno buscaba ante todo convencer y persuadir a la población de la necesidad de tener dos hijos como máximo, debía hacer frente al mismo tiempo a valores y creencias tradicionales (como la familia numerosa como cumbre de la felicidad). Estos últimos eran atacados continuamente por el Partido pero gozaban todavía de un apoyo importante entre la población. De manera aislada al principio y más constante con el paso del tiempo, las autoridades de Pekín empezaron a ejercer una presión social con el fin de garantizar el éxito de sus políticas antinatalistas. Por ejemplo, se prohibieron los beneficios de maternidad a partir del tercer hijo en las áreas urbanas. Asimismo, se recortaron los cupones para necesidades básicas a familias con más de dos hijos y se amenazó de expulsión de la universidad a los es-

46 Bongants, John y Greenhalgh, Susan, "An Alternative to the One-Child Policy in China”, Population and Development Review, 11, 4 (1985), págs. 585-617.

47 White, Tyrene, China's Longest Campaign..., pág. 60.

48 Peng Xizhao, Demographic Transition..., pág. 40. 
tudiantes que contrajeran matrimonio antes de finalizar los estudios. Un caso similar se dio en el caso de los aprendices en la formación profesional ${ }^{49}$. En las ciudades, donde las medidas de coacción eran mayores, la presión para la realización de una ligadura de trompa se hizo cada vez más fuerte para aquellas mujeres que tuvieran dos hijos. En las áreas rurales, la tolerancia era mayor pero los cuadros del Partido también ejercían en la medida de lo posible una fuerte coacción hacia las parejas que superaban los tres hijos.

Desde las altas esferas se recomendaba retrasar la edad de matrimonio, señalando que estos a una edad muy temprana solían ser perjudiciales, y se afirmaba que la edad ideal para casarse era entre los 25 y los 30 años, afirmándose que antes de los treinta se carecía de experiencia laboral y de recursos para llevar adelante una vida familiar adecuada ${ }^{50}$. De hecho, a partir de 1973 en algunas zonas se negaron a conceder licencias matrimoniales a hombres y mujeres menores de 30 y 28 años respectivamente. Otra medida fue convencer a las parejas de que tanto el hombre como la mujer eran responsables de la anticoncepción y de que los hombres debían cambiar su actitud respecto a la esterilización masculina. Ello iba acompañado de otra acción destinada a cambiar la mentalidad tradicional respecto al sexo de los hijos y convencer a las parejas de que las hijas eran igual de valiosas que los hijos varones.

Aquellas familias que optaban voluntariamente por tener dos o menos hijos eran favorecidas por el gobierno con mayores beneficios sociales y regalos, especialmente si uno de los cónyuges optaba por la esterilización. En este último caso incluso se podían obtener promociones laborables y, por tanto, incrementos de sueldo. Se impulsó el uso de anticonceptivos y se llevaron a cabo de manera gratuita tres tipos de operaciones: inserción de dispositivos intrauterinos (DIU), vasectomía y aborto, recompensado con entre una y tres semanas de baja laboral con sueldo según la importancia de las mismas ${ }^{51}$. No menos importante fue el auge de la píldora anticonceptiva a partir de esta época. El uso de anticonceptivos orales fue bien recibido por las parejas porque eliminaba los riesgos de una operación. Para las mujeres les evitaba someterse a la ligadura de trompas y para los hombres era una forma de evitar la esterilización, muy poco apreciada. Por otro lado, para las parejas era una forma de evitar embarazos no deseados y al mismo tiempo dejar la puerta abierta en caso de querer un nuevo embarazo en el futuro.

49 CHU, Leonard L., Planned Birth Campaigns in China..., págs. 68-71.

50 TiEn, H. Yuan, China's Population Struggle..., pág. 282.

51 La inserción del DIU conllevaba dos días de vacaciones; el aborto en el primer semestre, 14 días, al igual que la realización de un aborto durante el primer semestre acompañada de la inmediata inserción del DIU. Si tenía lugar un aborto durante el primer trimestre y era acompañado de una ligadura de trompas se recibía un mes de vacaciones (una cifra idéntica en caso de aborto durante el segundo semestre) mientras que un aborto durante el segundo semestre seguido de una ligadura de trompas era premiado con 40 días. La esterilización femenina recibía 21 días y la masculina (vasectomía), 7 días. Si una mujer optaba por esterilizarse justo después de dar a luz era premiada con 14 días de vacaciones que se añadían a los tres meses de baja materna. 
TABLA 2. MÉTODOS ANTICONCEPTIVOS EN CHINA (1971-1976)

\begin{tabular}{|c|c|c|c|c|}
\hline Año & $\begin{array}{c}\text { Inserción de } \\
\text { DIU }\end{array}$ & $\begin{array}{c}\text { Ligaduras de } \\
\text { trompa }\end{array}$ & Vasectomías & Abortos \\
\hline 1971 & 6.172 .889 & 1.744 .644 & 1.223 .480 & 3.910 .110 \\
1972 & 9.220 .297 & 2.087 .160 & 1.715 .822 & 4.813 .452 \\
1973 & 13.949 .569 & 2.955 .617 & 1.933 .210 & 5.110 .405 \\
1974 & 12.579 .886 & 2.275 .741 & 1.445 .251 & 4.984 .564 \\
1975 & 16.743 .693 & 3.280 .042 & 2.652 .653 & 5.084 .260 \\
1976 & 11.626 .510 & 2.707 .849 & 1.495 .540 & 4.742 .946 \\
\hline
\end{tabular}

Fuentes: Zhongguo weisheng nianjian 1985 [Anuario de Salud Pública, 1985], Pekín, Renmin weisheng chubanshe, 1986.

La principal característica de esta campaña de control de la natalidad será la obtención de los primeros éxitos tras el fracaso general de las anteriores. Aunque el crecimiento de la población seguirá a un ritmo similar a los de las décadas anteriores y en ocasiones se incrementará, ello será debido en mayor medida a hechos positivos: una reducción significativa de la tasa de mortalidad infantil y adulta (estimadas en un $45 \%$ y 7,25\%o en 1976 respectivamente) y el incremento de la esperanza de vida, en torno a 63-64 años a mediados de los setenta $^{52}$. Si analizamos los datos de la tabla 1 veremos que la campaña de 1970-1976 logró reducir de manera importante la tasa de natalidad, que pasó de 34,11\%o en 1969 a 19,91\%o, es decir, una disminución de casi 15 puntos en sólo siete años. Asimismo, no menos importante es la tendencia a la baja, fruto también de un cierto cambio social, de la tasa de fecundidad que pasó de 5,72 a 3,24 entre 1969 y 1976. Aunque la población china siguió creciendo, se habían puesto ya las bases para una política más audaz en lo que se refiere a la planificación familiar.

\section{CONCLUSIÓN}

En general, se puede afirmar que la era de Mao es un tiempo perdido en la lucha contra la sobrepoblación. El espectacular crecimiento que experimentó la $\mathrm{RPCh}$ en sus tres primeras décadas de existencia es uno de los pesados legados del maoísmo a la China de hoy pero conviene ser justos. Mao y sus camaradas del PCCh heredaron una China atrasada y pobre y donde las necesidades más urgentes eran asegurar la estabilidad y la paz social, el desarrollo económico y la mejora del nivel de vida de la gente. Es indudable que una de las causas de la explosión demográfica después de 1949 es la ausencia de políticas fuertes en lo que se refiere a la planificación familiar, al menos hasta la última etapa del

52 Banister, Judith, China's Changing Population..., pág. 352. 
maoísmo. Pero otra causa es también la rápida caída de la tasa de mortalidad, tanto adulta como infantil, gracias al desarrollo y la modernización de China. El régimen comunista impulsó medidas como la necesidad de higiene, el combate contra las enfermedades infeccionas (muchas de ellas hasta entonces endémicas), creó hospitales, clínicas y maternidades, se buscó convencer de dar a luz en dichos centros y no en el hogar y se difundieron técnicas modernas de alumbramiento ${ }^{53}$. Así, de una esperanza de vida media de 40 años en 1949 se pasó a una de 64 en 1976 mientras que la tasa de mortalidad infantil descendió de una cifra en torno a $200 \%$ a alrededor de $45 \%$ en el mismo periodo ${ }^{54}$.

Ahora bien, existe una responsabilidad clara por parte de Mao a la hora de no tomar medidas para frenar el crecimiento demográfico hasta la última etapa de su vida. Aunque el liderazgo en el seno del comunismo chino era compartido, Mao era de lejos la figura más importante y su acción determinaba el curso de las decisiones políticas y las políticas económicas y sociales. Sin ir más lejos, el apoyo que prestó en las tres campañas antinatalistas resultó fundamental para que éstas se pusieran en marcha seriamente. El problema es que él mismo, con su decisión de desencadenar el Gran Salto Adelante y más tarde la Revolución Cultural, afectó gravemente el desarrollo de los programas de planificación familiar hasta el punto de que sólo la tercera y última campaña tuvo un impacto verdadero en la sociedad y consiguió los primeros éxitos importantes. Dicho de otro modo, la labor política de Mao retrasó durante dos décadas la aplicación de unas medidas necesarias para combatir un fenómeno como era la sobrepoblación y sus consecuencias se prolongan hasta hoy.

De las tres campañas, la única que marcó un punto de inflexión en la lucha contra el elevado crecimiento demográfico fue la tercera (1971-1976) ${ }^{55}$. Pero las dos anteriores sentaron las bases de los éxitos de esta última y no debemos olvidar que se enfrentaron a problemas muy serios. Aparte de las resistencias dentro del Partido a todo cambio que limitara el número de nacimientos, la primera campaña (1954-1958) tuvo como enemigo principal los grandes pilares de la familia tradicional china, esto es, matrimonio joven, numerosos hijos y preferencia por el varón. Atacar estos pilares fue una de las acciones pero el impacto fue escaso. En la práctica, la primera campaña fue más propagandística que efectiva pero tuvo al menos una utilidad pues sirvió para que los dirigentes chinos se dieran cuenta de las dificultades y las complejidades de una política como la de planificación familiar. En este sentido, diseminó los prime-

53 Una tradición muy extendida hasta mediados del siglo XX era que la comadrona cortara con los dientes el cordón umbilical, con el riesgo evidente de infección para el recién nacido. Una causa de mortalidad infantil era la falta en muchas ocasiones de objetos no esterilizados.

54 BAnister, Judith, China's Changing Population..., pág. 352.

55 En realidad, esta tercera campaña no se detuvo en 1976 con la muerte de Mao sino que continuó y se intensificó en los años siguientes. Fue sustituida a partir de enero de 1979 por una todavía más firme, la campaña del «hijo único», que con diferentes matices sigue vigente hoy día en China. 
ros conocimientos efectivos sobre el control de la natalidad, enseñó que los objetivos debían cambiarse y estableció la necesidad de poner más medios para alcanzar el éxito en este ámbito. Entre las necesidades estaban nuevas técnicas de control de natalidad, aumentar el número de anticonceptivos al alcance de la población, más personal y más especializado, un incremento del presupuesto, una mayor y mejor organización y una mayor implicación del poder central, que había jugado un papel pasivo en esta campaña. La segunda campaña (1962-1966) recogió en gran medida estas necesidades pero fracasó principalmente porque no tuvo tiempo de consolidarse. No obstante, demostró que un mayor control del gobierno y el apoyo de las provincias podía conseguir éxitos (circunscritos lamentablemente sólo al ámbito urbano). Esta campaña también demostró claramente que a ojos de las autoridades chinas el tener hijos no era sólo una cuestión personal y exclusiva de la pareja sino también social, política y económica porque el Estado tenía que proporcionar comida, ropa, educación, transporte, empleo, sanidad, etc. para los hijos a medida que crecían. No es extraño, por tanto, el intervencionismo estatal en ese asunto. Pese a sus fracasos, las dos primeras campañas también tuvieron un impacto decisivo a la larga pues su aplicación permitió derogar leyes que impedían el avance de estas políticas antinatalistas (legalización de importación de anticonceptivos, levantamiento de la prohibición casi total que existía sobre el aborto desde 1949, etc.) y reducir la influencia de los opositores al control demográfico que había en el seno del régimen comunista.

La tercera campaña, heredera de las anteriores, es fundamental en la historia de la política de planificación familiar de China. En primer lugar porque es la primera en lograr un éxito significativo en la lucha contra la superpoblación. En segundo lugar porque dicha campaña es la base sobre la que se asentaría la política antinatalista del régimen chino a partir de 1979 y que le haría famoso en todo el mundo: la política del «hijo único». La tercera campaña fue importante también porque conllevó la aceptación de la planificación familiar por una parte importante de la población china. A ello no fueron ajenos los cambios en el estatus y el papel de la mujer en la sociedad y en la familia china, los cambios sociales y económicos y la mejora del nivel de vida general. No menos importante fue la labor del Estado y su política de control, que no dudó en enfrentarse de lleno a la resistencia social y atacar los valores tradicionales. La base de su éxito fue, en este sentido, la coacción pese a que las autoridades siempre la habían considerado contraproducente y poco exitosa. En la primera campaña no hubo coacción porque todavía el gran número de nacimientos no era visto como una amenaza para el desarrollo económico y la estabilidad social mientras que en la segunda se aplicaron las primeras medidas de castigo aunque de manera muy restringida y en general se limitaban más a la amenaza que al cumplimiento de las sanciones. Pero en los años setenta, al convertirse la planificación familiar en un objetivo de la revolución y de la construcción del socialismo, toda resistencia a aquella fue considerada una oposición al Partido y por ello se implantó un sistema de penalizaciones y recompensas. En la primera 
campaña no hubo un límite en el número de nacimientos mientras que en la segunda existió una recomendación de no pasar del tercer hijo. En cambio, en la tercera campaña se implantó ya un sistema de presión social y económica sobre las parejas para que evitaran tener más de dos hijos. El único aspecto en que fracasó el Estado fue en su objetivo de regularizar la edad de matrimonio porque se trataba de un aspecto muy impopular y suponía a la larga muchos problemas en una sociedad tan conservadora como la china (cohabitación y relaciones sexuales prematrimoniales, embarazos ilegítimos, etc. ${ }^{56}$. En la práctica, los cambios sociales y económicos llevaron a las parejas, especialmente en las ciudades, a retrasar progresivamente y de manera voluntaria la edad de matrimonio.

En definitiva, la tercera campaña demostró que la definición clara y realista de los objetivos, mejores campañas de información, el suministro de más y nuevos anticonceptivos (píldora), una mejora del sistema sanitario en lo que se refiere a interrupción del embarazo, la formación de más personal y mejor cualificado, mejor organización y mayores fondos eran fundamentales para que la política de planificación familiar saliera adelante. Aparte, el éxito no hubiera tenido lugar sin la colaboración de las parejas, que ya fuera por fuerza o voluntariamente aceptaron y reconocieron que limitar su descendencia iba en beneficio suyo. Por último, un factor decisivo fue la coacción y la aplicación de medidas de castigo y de recompensa. Todos estos elementos serían recogidos por el régimen comunista chino tras la muerte de Mao y se aplicarían con toda su fuerza hasta nuestros días.

Recibido: 21-09-2009

Aceptado: 30-06-2010

56 La Ley del Matrimonio de 1950, que establecía la edad mínima para casarse en 20 años para los hombres y 18 en el caso de las mujeres, nunca fue revocada durante esta época. 